\title{
Conditioning of simultaneous and successive common elements in a discrimination and pseudodiscrimination
}

\author{
HEIDI UDELL and ROBERT A. RESCORLA \\ Yale University, New Haven, Connecticut 06520
}

\begin{abstract}
In an autoshaping procedure, pigeons received concurrent exposure to both a discrimination and a pseudodiscrimination treatment with color-line orientation compounds. One color was presented with the orientations when they were differentially reinforced; the other color was presented when the same orientations were given in a pseudodiscrimination procedure. Subsequently, responding was assessed to the separately presented colors. When the elements of the color-orientation compounds had been presented simultaneously, the color from the discrimination received less conditioning than did that from the pseudodiscrimination. However, when the color preceded the orientations during training, the color from the discrimination treatment became more conditioned than did that from the pseudodiscrimination. Although reconciling some apparent discrepancies from previous studies, these results also point to an incompleteness in current theories of discrimination learning.
\end{abstract}

It has become a frequent theme in studies of simple learning processes that the information that a stimulus provides about important events determines the learned value of that stimulus. Especially in the study of Pavlovian conditioning, it is popular to assert that associations are formed between a conditioned stimulus (CS) and an unconditioned stimulus (US) to the degree that the former provides new information about the occurrence of the latter (e.g., Mackintosh, 1974; Rescorla, 1972).

One outcome that has encouraged this view is well illustrated by the experiments of Wagner, Logan, Haberlandt, and Price (1968). In those experiments, two groups of animals were exposed to a particular stimulus, $\mathrm{X}$, which was reinforced on half of its occurrences. For one group, that exposure was embedded in an $\mathrm{AX}+\mathrm{BX}-$ discrimination procedure, in which A always accompanied $\mathrm{X}$ on its reinforced occasions and $\mathrm{B}$ always accompanied $X$ when it signaled nonreinforcement. A second group also received AX and BX presentations, but each was reinforced on a random half of its occurrences. This group received a pseudodiscrimination of the form $\mathrm{AX} \pm / \mathrm{BX} \pm$. Although the reinforcement schedules for $\mathrm{X}$ were similar in both groups, they differed in the degree to which $\mathrm{A}$ and $\mathrm{B}$ provided information about that reinforcement. In the first group, A and B were well correlated with reinforcement; in the second group, they were no more correlated with reinforcement than was X. As expected from the fact that these treatments resulted in its being relatively less informative in the first group, $\mathrm{X}$ became substantially less conditioned in that group.

This research was supported by NSF Grant BNS-78-02752. Requests for reprints should be sent to R. A. Rescorla, Box 11a Yale Station, New Haven, Connecticut 06520.
Another source of encouragement for a role of information in conditioning is studies attempting to identify a reinforcing value for information itself. For instance, Prokasy (1956) offered rats the choice between two goalboxes in a $\mathrm{T}$ maze, both of which contained food on a $50 \%$ schedule. However, on one side of the maze, the brightness of a delay chamber and the goalbox signaled whether food would occur on a trial; on the other side of the maze, the same delay chamber and goalbox brightnesses were also given, but in a manner uninformative with regard to the occurrence of food reinforcement. Prokasy found that the rats preferred the side on which the brightnesses were informative, despite the equivalence of food reinforcement on the two sides. One interpretation of this finding is that information per se is reinforcing.

It is interesting to note that one may describe Prokasy's (1956) procedure in terminology analogous to that of Wagner et al. (1968). Let X and Y stand for the stimuli between which the rat chooses at the choice point of the T maze, and let $A$ and $B$ represent the different brightnesses of the delay chamber and goalboxes. Then, on the informative side of the maze, the rat receives an $\mathrm{AX}+/ \mathrm{BX}$ - procedure in which the choicepoint stimulus, $X$ is reinforced when followed by $A$ but nonreinforced when followed by $B$. On the other side of the maze, the rat receives an $A Y \pm / B Y \pm$ procedure in which the $Y$ choice-point stimulus is reinforced half the time whether followed by A or B. In the language of this description, the outcome is that the rat chooses $\mathrm{X}$ over $\mathrm{Y}$. If one accepts that choice as evidence that $\mathrm{X}$ is better conditioned by food than is $\mathrm{Y}$, then the result of Prokasy's experiment is that a common element embedded in a discrimination procedure became better conditioned than did one embedded 
in a pseudodiscrimination. That is, when described in formal terms, the outcome of Prokasy's experiment is the opposite of that reported by Wagner et al. Although both outcomes have been interpreted as suggesting an informational approach to learning, they are in fact empirically opposite.

There are, of course, many ways in which these experiments differ from each other. For instance, they differ in both the kind of apparatus and the measure of learning. However, two differences are of special interest in the present report. The first is the contrast between a within- and between-subjects design. The animals of the Wagner et al. (1968) study were exposed only to one kind of treatment, whereas Prokasy's (1956) animals were required to learn both a discrimination and a pseudodiscrimination with partially overlapping stimuli. Such a difference in task complexity might well encourage different processes. Indeed, as noted below, the major accounts of the Wagner et al. findings do not anticipate similar results when the same subject must learn both a discrimination and a pseudodiscrimination. A second difference is in the temporal pattern of event presentation within a trial. In the Wagner et al. study, $\mathrm{X}$ was presented concurrently with the $\mathrm{A}$ and $\mathrm{B}$ stimuli, whereas in the Prokasy study, $\mathrm{X}$ and $\mathrm{Y}$ were presented prior to $\mathrm{A}$ and $\mathrm{B}$. It seems possible that sequential stimulus presentation might lead to the organism's treating $\mathrm{X}$ and $\mathrm{Y}$ in a different manner. For instance, with sequential presentation, $A$ and $B$ may serve as reinforcers for $\mathrm{X}$ and $\mathrm{Y}$, thus providing a source other than the US for their conditioning.

Consequently, the present study examined the role of those two variables. It first replicated the outcome of the Wagner et al. (1968) study with simultaneous stimulus presentation, but using a within-subjects design. It then found those results to be reversed when the mode of stimulus presentation was made sequential. Because of the multiplicity of stimuli needed for such a study, an autoshaping procedure was used with pigeon subjects. Wasserman (1974) has demonstrated that the results of the Wagner et al. study are sufficiently robust to appear in that preparation.

\section{METHOD}

\section{Subjects and Apparatus}

The subjects were 16 adult female Carneaux pigeons, maintained at $75 \%$ of their free-feeding body weights. These birds had previously been subjects in several autoshaping experiments, none of which employed the stimuli of the present experiment.

The apparatus comprised eight identical Skinner boxes, $27 \times 27 \times 35 \mathrm{~cm}$. The metal front panel of each box had a $5 \times 5 \mathrm{~cm}$ food magazine in its center, located $5 \mathrm{~cm}$ from the wire-mesh floor. Three response keys, $2.5 \mathrm{~cm}$ in diameter, were located one directly above the hopper and one $5 \mathrm{~cm}$ on either side of the center of the front wall, $20 \mathrm{~cm}$ above the floor. Only the left-hand key was operative in the present experiment. The remaining walls and ceiling were clear Plexiglas. Stimuli were presented by an IEE in-line projector, located behind the key. This projector contained a slide that permitted separate illuminations of the right and left sectors of the key. The left sector could be illuminated by either a red $(R)$ or a green $(G)$ light; the right sector was illuminated by a grid of 1-mm black lines on a white background that was either horizontal $(\mathrm{H})$ or vertical (V) in orientation. When either half of the key was separately illuminated, the other half remained dark; when both sides were simultaneously illuminated a $1-\mathrm{mm}$ black vertical line separated the two components.

The Skinner boxes were placed inside sound- and lightattenuating chambers, with ventilation fans providing background noise. These chambers had a $6-\mathrm{W}$ bulb mounted on the rear wall that was continuously illuminated during the session except during the operation of the food hopper. That hopper contained Purina Pigeon Grain.

\section{Procedure}

Because of their experimental histories, the pigeons required no magazine training; instead, discriminative autoshaping was begun immediately. All animals were initially exposed to a discrimination procedure of the form $\mathrm{AX}+, \mathrm{BX}-, \mathrm{AY} \pm, \mathrm{BY} \pm$, in which " + " and "-" stand for reinforcement and nonreinforcement, respectively. For all animals, A and B were, respectively, the horizontal and vertical grids; for half the animals, $\mathrm{X}$ was the green light and $\mathrm{Y}$ was the red light; for the remaining half, the colors were interchanged.

During each of 12 sessions, the birds received 12 presentations of each trial type. All CS presentations consisted of $5 \mathrm{sec}$ of simultaneous illumination of the key by a color-orientation compound. Where appropriate, the trial terminated in the 5 -sec availability of grain from the hopper. The intertrial interval was variable around a mean of $1 \mathrm{~min}$.

On the next day, a single test session assessed the responding to separate presentations of $X$ and $Y$ (i.e., $R$ and $G$ ). That session began with continued discrimination training in the form of six presentations of each of the appropriate four trial types. Then each animal received six nonreinforced presentations of $R$ and G, presented separately for 5 sec.

Following this test session, 25 additional sessions of discrimination training were given. These sessions were identical to those described above, except that the presentation of the stimuli was sequential within a trial. Each 5-sec presentation of $\mathrm{X}$ or $\mathrm{Y}$ was followed by a 5-sec presentation of $\mathrm{A}$ or $\mathrm{B}$ and then the appropriate reinforcement. Twelve presentations of each type of trial were given; the intertrial interval remained $1 \mathrm{~min}$.

On the next day, another test session was given; that session was identical to the first test session except that the initial discrimination trials maintained the sequential, rather than simultaneous, stimulus presentations.

\section{RESULTS AND DISCUSSION}

Despite its complexity, the discrimination proved reasonably easy for the birds to learn. The left-hand panel of Figure 1 shows the rate of pecking on the various trial types for the last day of the initial discrimination training phase. It is quite clear that the response rate during $\mathrm{BX}$ - trials was substantially lower than that on $\mathrm{AX}+$ trials. Every animal showed that discrimination. The high rates of responding on the pseudodiscrimination trials, AY and BY, are typical of such procedures. Those rates differed neither among themselves nor from the rate during $\mathrm{AX}$.

The second panel of Figure 1 shows the results of separate testing of $\mathrm{Y}$ and $\mathrm{X}$ on the first test session. Responding was substantially higher during $\mathrm{Y}$ (Wilcoxon $\mathrm{T}=11, \mathrm{p}<.001)$. That outcome replicates, using a within-subjects design, the results of Wagner et al. (1968) and Wasserman (1974). The amount of conditioning to a common element in a discrimination was 


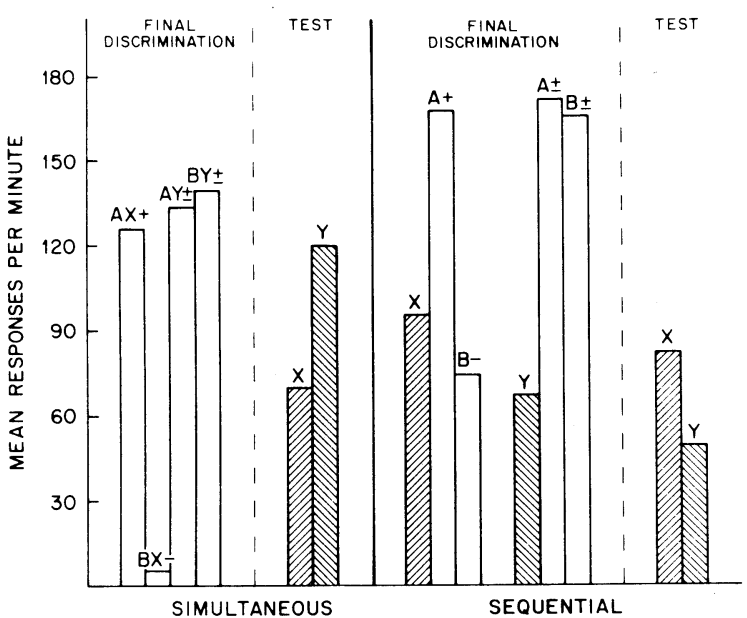

Figure 1. Rate of responding to the compound discriminanda during discrimination training and to the common elements ( $X$ and $Y$ ) in the tests. $A$ and $B$ were horizontal and vertical black grids on white backgrounds; $X$ and $Y$ were red and green colors. All animals learned both a discrimination $(\mathbf{A X}+/ \mathbf{B X}-)$ and a pseudodiscrimination $(\mathrm{AY} \pm / \mathrm{BY} \pm)$ in which the elements were presented either simultaneously or successively.

lower than that to a common element in a comparable pseudodiscrimination.

The subsequent acquisition of the discrimination when $\mathrm{X}$ and $\mathrm{Y}$ preceded $\mathrm{A}$ and $\mathrm{B}$ proved substantially more extended. Initially, the birds responded at a high rate to both components of all trials. However, by the end of 25 sessions, a sufficient discrimination emerged to allow an assessment of the strengths of the individual elements. The third panel of Figure 1 shows the performance to various stimuli on the final discrimination session of this phase. Note first that the pattern of performance during the $\mathrm{A}$ and $\mathrm{B}$ stimuli is like that during those stimuli in the first phase of the experiment. When they were preceded by $\mathrm{X}$, responding to $A$ and $B$ was differential (Wilcoxon $T=0, p<.001$ ); however, when they were preceded by $\mathrm{Y}$, responding occurred at an equivalent rate to the $\mathrm{A}$ and $\mathrm{B}$ stimuli.

Of more interest, the responding to $\mathrm{X}$ and $\mathrm{Y}$ that could be observed during their sequential presentations also proved to be differential. By the end of this phase of discrimination training, responding to $\mathrm{X}$ was reliably greater than that to $\mathrm{Y}$ (Wilcoxon $\mathrm{T}=5, \mathrm{p}<.001$ ). The differential responding that was observed in the course of discrimination trials was confirmed in the subsequent test session. During that session, nonreinforced presentations of $\mathrm{X}$ also elicited a higher pecking rate than did those of $Y(T=1.5, p<.001)$. Thus, the results of the sequential presentation agree with those of Prokasy (1956); with sequential presentation, responding was greater to the common element of a discrimination than to that of a pseudodiscrimination.

Although the results are not displayed here, the animals were subsequently returned to a simultaneous discrimination procedure; the consequence of that return was the removal of any difference in responding to $\mathrm{X}$ and $\mathrm{Y}$.

These results suggest that an important contributor to the relative strengths of common elements in pseudodiscrimination and real discrimination is the temporal relation to potential discriminative elements. With simultaneous presentation, the common elements receive less conditioning than do more informative stimuli. However, with sequential presentation, common elements become more conditioned when followed by informative rather than noninformative stimuli.

These results have several implications for the interpretation of the outcomes of discrimination learning experiments. There are two principal accounts of the Wagner et al. (1968) finding of greater conditioning of a simultaneous common element in a pseudodiscrimination. However, neither of those accounts anticipates the easy solution to the within-subjects discrimination of the present experiment. One account (Sutherland \& Mackintosh, 1971) emphasizes the role of differential attention to the various stimuli. According to that account, $\mathrm{A}$ and $\mathrm{B}$ receive substantial attention in the discrimination because of their high correlation with reinforcement. As a consequence, $\mathrm{X}$ receives less attention and so less conditioning than in a pseudodiscrimination in which A and B are less well correlated with reinforcement. However, in the discrimination procedure of the present experiment, the animal cannot reduce its attention to $\mathrm{X}$ (or $\mathrm{Y}$ ) and still respond appropriately. Because A and B are both correlated and uncorrelated with reinforcement, depending upon whether they are accompanied by $\mathrm{X}$ or $\mathrm{Y}$, the organism must attend to $\mathrm{X}$ and $\mathrm{Y}$ in order to appropriately show differential or nondifferential behavior to $\mathrm{A}$ and $\mathrm{B}$.

A second account (Rescorla \& Wagner, 1972) instead derives the lesser conditioning of $\mathrm{X}$ in a discrimination paradigm from the greater conditioning of $A$ and, hence, its ability to block conditioning of $\mathrm{X}$ on $\mathrm{AX}+$ trials. However, that account also anticipates that the use of the same stimuli in both a pseudodiscrimination and a discrimination procedure should prevent the development of appropriately differential behavior.

This observation suggests the need for a different account of the responding to the common elements of a discrimination and a pseudodiscrimination. One potential candidate is "configural conditioning," in which each of the four stimuli is treated as a separate entity not adequately described in terms of its component parts. However, such a view fails to anticipate any specific differential behavior to $\mathrm{X}$ and $\mathrm{Y}$ similar to that observed in the present experiment. Alternatively, one might adopt a hierarchical view of the operation of the various stimulus components. It may be that the role of $\mathrm{X}$ and $\mathrm{Y}$ is not to enter into performance in a manner comparable to $\mathrm{A}$ and $\mathrm{B}$, but, rather, is to direct the organism as to how to treat $\mathrm{A}$ and $\mathrm{B}$. 
The present findings of superior performance to a sequential common element in a pseudodiscrimination can be viewed as consistent with Mackintosh's (1974) analysis of such situations. The use of sequential presentation may permit not only the ultimate food reinforcement, but also the A and B stimuli to serve as reinforcers for $\mathrm{X}$ and $\mathrm{Y}$. In that case, the total reinforcement to be gained from an A and B that are well correlated with the US may be greater than that to be gained from two stimuli that are each partially correlated with that US However, in the present case, it is important to note that the same A and B follow both $\mathrm{X}$ and $\mathrm{Y}$. Consequently, if $\mathrm{A}$ and $\mathrm{B}$ have differential reinforcement power for $\mathrm{X}$ and $\mathrm{Y}$, it can only be because $\mathrm{X}$ and $\mathrm{Y}$ signal their correlation with the ultimate reinforcer.

Whatever the value of these speculations, the present results point to the importance of sequential vs. simultaneous stimulus presentation as a determinant of learning about common elements in a discrimination. They clearly seem to require an expansion of our current theories of such effects.

\section{REFERENCES}

Mackintosh, N. J. The psychology of animal learning. New York: Academic Press, 1974.

Prokasy, W. F. The acquisition of observing responses in the absence of differential external reinforcement. Journal of Comparative and Physiological Psychology, 1956, 49, 131-134.

RESCORLA, R. A. Informational variables in Pavlovian conditioning. In G. H. Bower (Ed.), The psychology of learning and motivation (Vol. 6). New York: Academic Press, 1972.

Rescorla, R. A., \& Wagner, A. R. A theory of Pavlovian conditioning: Variations in the effectiveness of reinforcement and nonreinforcement. In A. H. Black \& W. F. Prokasy (Eds.), Classical conditioning II: Current Research and Theory. New York: Appleton-Century-Crofts, 1972.

Sutherland, N. S., \& Mackintosh, N. J. Mechanisms of animal discrimination learning. New York: Academic Press, 1971.

Wagner, A. R., Logan, F. A., Haberlandt, K., \& Price, T. Stimulus selection in animal discrimination learning. Journal of Experimental Psychology, 1968, 76, 171-180.

Wasserman, E. A. Stimulus-reinforcer predictiveness and selective discrimination learning in pigeons. Journal of Experimental Psychology, 1974, 103, 284-297.

(Received for publication August 28, 1979.) 